\title{
In-ring velocity measurement for isochronous mass spectrometry
}

\author{
X. Zhou $\odot,{ }^{1,2}$ M. Zhang, ${ }^{1,2}$ M. Wang, ${ }^{1,2, *}$ Y. H. Zhang, ${ }^{1,2, \dagger}$ Y. J. Yuan, ${ }^{1,2, \sharp}$ X. L. Yan, ${ }^{1, \S}$ \\ X. H. Zhou, ${ }^{1,2}$ H. S. Xu ${ }^{1,2}$ X. C. Chen, ${ }^{1}$ Y. M. Xing, ${ }^{1}{ }^{1,}$. J. Chen, ${ }^{1,3}$ X. Xu ${ }^{4,1}$ P. Shuai, ${ }^{1}$ \\ C. Y. Fu, ${ }^{1}$ Q. Zeng, ${ }^{1,5}$ M. Z. Sun, ${ }^{1}$ H. F. Li, ${ }^{1,2}$ Q. Wang, ${ }^{1,2}$ T. Bao, ${ }^{1}$ M. Si ${ }^{1,2,6}$ H. Y. Deng, ${ }^{1,2}$ \\ M. Z. Liu, ${ }^{1,2}$ T. Liao, ${ }^{1,2}$ J. Y. Shi, ${ }^{1,2}$ Y. N. Song, ${ }^{1,2}$ J. C. Yang, ${ }^{1}$ W. W. Ge, ${ }^{1}$ Yu. A. Litvinov, ${ }^{1,3}$ \\ S. A. Litvinov, ${ }^{1,3}$ R. S. Sidhu, ${ }^{3}$ T. Yamaguchi, ${ }^{7}$ S. Omika, ${ }^{7}$ K. Wakayama, \\ S. Suzuki, ${ }^{1,8}$ and T. Moriguchi ${ }^{8}$ \\ ${ }^{1}$ CAS Key Laboratory of High Precision Nuclear Spectroscopy, Institute of Modern Physics, \\ Chinese Academy of Sciences, Lanzhou 730000, China \\ ${ }^{2}$ School of Nuclear Science and Technology, University of Chinese Academy of Sciences, \\ Beijing 100049, China \\ ${ }^{3}$ GSI Helmholtzzentrum für Schwerionenforschung, Planckstraße 1, 64291 Darmstadt, Germany \\ ${ }^{4}$ School of Physics, Xi'an Jiaotong University, Xi'an 710049, China \\ ${ }^{5}$ School of Nuclear Science and Engineering, East China University of Technology, \\ Nanchang 330013, China \\ ${ }^{6}$ Université Paris-Saclay, CNRS/IN2P3, IJCLab, F-91405 Orsay, France \\ ${ }^{7}$ Department of Physics, Saitama University, Saitama 338-8570, Japan \\ ${ }^{8}$ Institute of Physics, University of Tsukuba, Ibaraki 305-8571, Japan
}

(Received 28 September 2020; accepted 17 March 2021; published 14 April 2021)

\begin{abstract}
Isochronous mass spectrometry based on heavy-ion storage rings is a powerful tool for direct mass measurements of very short-lived nuclei. Owing to the nature of in-flight separation of high-energy reaction products, many ion species with different mass-to-charge ratios $(\mathrm{m} / \mathrm{q})$ can be transmitted to and stored in the ring in one shot. However, high mass resolving power can be achieved only for a limited range of ion species with velocities well matching the isochronous condition of the ring. The knowledge of velocities of each stored ions is required to overcome this restriction. For this purpose, two time-of-flight (TOF) detectors were installed $18 \mathrm{~m}$ apart in one straight section of the cooler-storage ring CSRe in Lanzhou. The time sequences measured by the two TOF detectors for each stored ion were used for the precision determination of its velocity. A relative precision of the velocity is achieved to the level of $10^{-5}$ for individual ions. The betatron oscillations of the ion motion in the ring were clearly identified in the data and were taken into account in the analysis. The presented technique can be used for measurements of machine tunes and their dependence on particle momenta.
\end{abstract}

DOI: 10.1103/PhysRevAccelBeams.24.042802

\section{INTRODUCTION}

Isochronous mass spectrometry (IMS) at high-energy storage ring facilities is a powerful tool for measuring

\footnotetext{
*Corresponding author. wangm@impcas.ac.cn

Corresponding author.

yhzhang@impcas.ac.cn

Corresponding author. yuanyj@impcas.ac.cn

${ }^{\S}$ Current address : Argonne National Laboratory, 9700 South Cass Avenue, Lemont, Illinois 60439, USA.

Published by the American Physical Society under the terms of the Creative Commons Attribution 4.0 International license. Further distribution of this work must maintain attribution to the author(s) and the published article's title, journal citation, and DOI.
}

masses of short-lived nuclei produced via projectile fragmentation or induced fission reactions [1-3]. Since the pioneering work at the experimental storage ring (ESR) at GSI in Darmstadt [4,5], IMS has been established at the cooler storage ring (CSRe) at the Institute of Modern Physics (IMP) in Lanzhou [6,7], and is under development at the Rare RI Ring (R3) at RIKEN in Saitama $[8,9]$.

By using IMS at the CSRe, masses of more than 30 short-lived nuclei have been measured in the past few years [10-19]. In these experiments, nuclei of interest were stored in the CSRe and their revolution times were measured by using a single time-of-flight (TOF) detector $[20,21]$. A part of the TOF detector is a thin foil installed in the ring aperture. Ions traversing through the foil at each revolution provide fast timing signals used to extract their mean revolution times. Many different nuclear reaction products are studied in one experiment. Mean revolution 
times of the stored ions, $T$, depend on their mass over charge ratios, $m / q$, as $[4,22]$

$$
\frac{\Delta T}{T}=\frac{1}{\gamma_{t}^{2}} \frac{\Delta(m / q)}{(m / q)}+\frac{\delta T}{T},
$$

where $\gamma_{t}$ is the so-called transition energy of the storage ring. The term $\delta T / T$ represents the variable resolving power. By using Eq. (1) and revolution times of ions with well-known masses, a calibration curve is established which is then used to determine previously unknown nuclear masses [4,23].

For each ion species stored in the ring, the spread of revolution times $T$ is determined by the magnetic rigidity $(B \rho)$ acceptance of the ion optical system as $[24,25]$

$$
\frac{\delta T}{T}=\left(\frac{1}{\gamma_{t}^{2}}-\frac{1}{\gamma^{2}}\right) \frac{\delta(B \rho)}{B \rho}=\left(\frac{\gamma^{2}}{\gamma_{t}^{2}}-1\right) \frac{\delta v}{v},
$$

where $\gamma$ is the mean relativistic Lorentz factor of the stored ion species and $v$ is the velocity of the ion. In the case of the CSRe, $\gamma_{t}$ can be varied from 1.3 to 1.4 depending on the ring settings $[26,27]$. The magnetic rigidity of a single ion is determined by $B \rho=p / q=m / q \cdot v \gamma$, with $p$ being the momentum of the ion.

Right after the production target, the momentum spread of the reaction products is much larger than the typical acceptance of a storage ring [22]. Ion species with different velocities but matching the magnetic rigidity can be transmitted to and stored in the ring. From Eq. (2), it is obvious that high time resolutions (equivalent to the high mass resolving powers) can be achieved for ion species that well match the isochronous condition of $\gamma=\gamma_{t}$ [4]. For ion species with $\gamma$ differing from $\gamma_{t}$, the isochronous condition is not well matched, resulting in a broadening of the corresponding revolution-time distribution. Furthermore, the distributions can become asymmetric due to asymmetric momentum spread at the injection into the ring [28]. As a result, the mass resolving power is quickly decreasing when moving away from the isochronous condition.

It was recognized years ago that the velocity of each stored ion is needed to correct for nonisochronicity effects $[29,30]$. This has been laterally verified in the ESR [31] as well as in the CSRe [32] by limiting the momentum spread of the ions with mechanical slits, though at a cost of dramatically reduced transmission efficiency. The velocity measurements can be conducted by using two TOF detectors either outside or inside the ring. For example, in the IMS experiments at the R3 in RIKEN, the velocity of each ion is measured by two timing detectors installed $84 \mathrm{~m}$ apart in the fragment separator before injection into the R3 ring. A relative precision of $\sim 10^{-4}$ is expected there [8]. The driver accelerator in RIKEN is a superconducting cyclotron providing quasi-direct-current primary beam. Such approach is possible at the R3 since only a single ion identified in the fragment separator is injected at a time. It is different at GSI and IMP facilities, where the fast extraction of the pulsed primary beams of a synchrotron onto the target produces a bunch of reaction products with a pulse duration of $0.3-0.5 \mu \mathrm{s}$. For dozens of particles in a single bunch, it is not feasible to perform particle-byparticle velocity measurements in the fragment separator in coincidence with the revolution times measured in the ring. In the past decade, the possibility has been investigated to perform simultaneous measurements of the revolution times as well as the velocities of stored ions [24,30]. In this work we report the first precision in-ring velocity measurements of ions stored in the CSRe.

\section{EXPERIMENT}

The experiment was conducted at the Institute of Modern Physics in Lanzhou. The ${ }^{58} \mathrm{Ni}^{19+}$ primary beams with an energy of $440 \mathrm{MeV} / \mathrm{u}$ were fast extracted from the heavy ion synchrotron the main Cooler-Storage-Ring and guided to a $\sim 15 \mathrm{~mm}$ thick ${ }^{9} \mathrm{Be}$ target. The projectile fragments of interest were selected in flight by the radioactive ion beam line (RIBLL2) before they were injected into the CSRe. Injections were repeated every few ten seconds.

The CSRe was tuned in the isochronous mode with $\gamma_{t}=1.365$. The momentum acceptance of the CSRe is $\pm 0.2 \%$. Figure 1 shows calculated betatron amplitude functions $\beta_{x, y}$ and dispersion functions $D_{x, y}$ along the reference closed orbit in the CSRe, $s$. These functions are used to determine the closed orbit and betatron oscillation amplitudes for a stored particle with a certain momentum and position deviation from the reference particle $[33,34]$. The RIBLL2-CSRe system was set to a fixed central magnetic rigidity of $B \rho=5.471 \mathrm{Tm}$. In this setting, the isochronous condition was well matched for the nuclei with mass-to-charge ratio $A / Z \approx 1.9$ (around ${ }^{53} \mathrm{Ni}^{28+}$

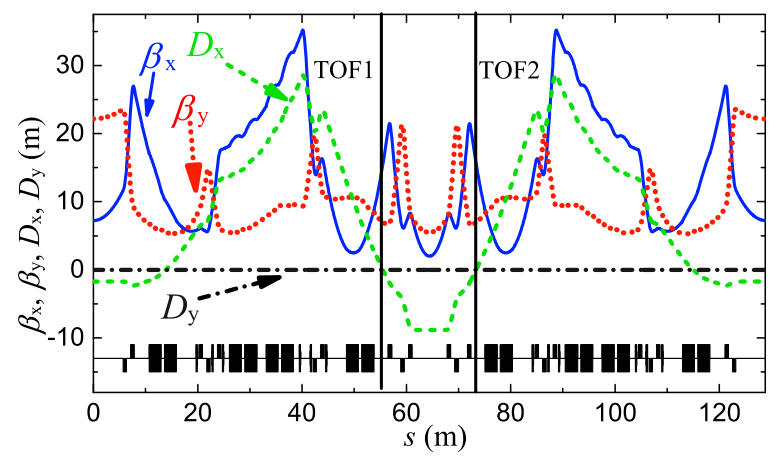

FIG. 1. Calculated horizontal, $\beta_{x}(s)$ (blue solid line), and vertical, $\beta_{y}(s)$ (red dotted line), betatron amplitude functions and the horizontal, $D_{x}(s)$ (green dashed line), and vertical, $D_{y}(s)$ (black dashed and dotted line) dispersion functions of the CSRe lattice employed in this experiment. Calculations were done with WINAGILE code [35]. The positions of the two TOF detectors are marked by black solid vertical line. 
and ${ }^{55} \mathrm{Cu}^{29+}$ ), which had an optimum transmission efficiency through the system as well.

Two TOF detectors, TOF1 and TOF2, were installed $18 \mathrm{~m}$ apart in one of the straight sections of the CSRe as shown in Fig. 1 [24,36-38]. The diameter of the carbon foil in the TOF detector is only $40 \mathrm{~mm}$, which limited the horizontal acceptance to $\sim 20 \pi \mathrm{mm}$ mrad. Each ion circulating in the ring successively passed through TOF1 and TOF2 at each revolution and could produce timing signals in both detectors. These timing signals were recorded by the same digital oscilloscope via two input channels. The sampling rate was $50 \mathrm{GHz}$. The acquisition time was $400 \mu \mathrm{s}$ after the injection trigger, corresponding to about 600 revolutions of the ions in the ring. From the recorded timing signals, the time stamps of each ion species [23], $t_{\exp }(N)$, i.e., the absolute passing times of the ions at the revolution number $N$ after injection, are extracted using the constant fractional discrimination technique. In this experiment, about ten ions of different $\mathrm{Z}$ were stored in the ring in each injection, and the fraction of one specific ion species depends mainly on its production yield and transmission efficiency. The particle identifications were easily made according to the procedures described in Refs. [23,39].

Figure 2 shows a pair of time sequences of a single ${ }^{45} \mathrm{~V}^{23+}$ ion from the two detectors. As can be seen in the inset of this figure, a passage of the ion through the detector did not always produce a time stamp. This is because the detection efficiencies varied from $10 \%$ to $90 \%$ depending on the atomic number $Z$ of the ion as well as on the total number of stored ions [20,21]. For the time sequences in Fig. 2, 437 and 441 time stamps were recorded by the

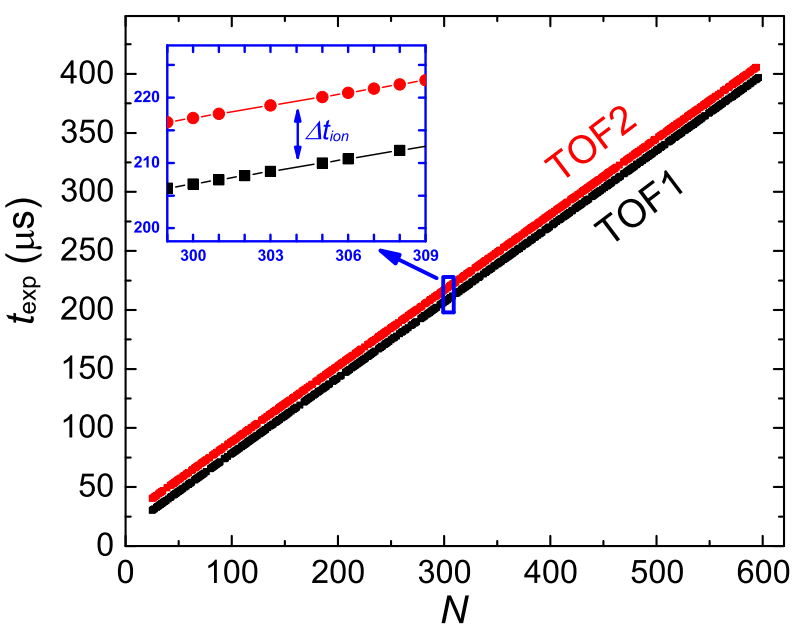

FIG. 2. Time sequences of a single ${ }^{45} \mathrm{~V}^{23+}$ ion measured by TOF1 and TOF2 detectors as a function of the revolution number $N$. For illustration purpose, the time stamps from TOF2 are shifted upwards by $10 \mu \mathrm{s}$. The inset illustrates a region zoomed at $299 \leq N \leq 309$ showing the missing time stamps in some revolutions. The error bar of each data point is within the symbol size.
TOF1 and TOF2 detectors, respectively, from the overall 570 revolutions in the ring. This corresponds to the average detection efficiencies of $76.6 \%$ (TOF1 detector) and $77.4 \%$ (TOF2 detector) for this ${ }^{45} \mathrm{~V}^{23+}$ ion. For the data shown in Fig. 2, 342 one-to-one coincident time stamps were identified. In the following, the data presented in Fig. 2 are used as an illustrative example.

\section{VELOCITY DETERMINATION}

The mean velocity of an ion circulating in the ring, $v$, can be obtained via

$$
v=\frac{L}{\Delta t_{\mathrm{ion}}}=\frac{L}{\Delta t_{\mathrm{exp}}-\Delta t_{d}},
$$

with $L$ being the distance between the two TOF detectors in the straight section of CSRe, $\Delta t_{\text {ion }}$ the mean time-of-flight of the ion between the two detectors, $\Delta t_{\exp }$ is the mean time-offlight extracted from the measured data, and $\Delta t_{d}=t_{d}^{\mathrm{TOF} 2}-$ $t_{d}^{\mathrm{TOF} 1}$ the time delay difference between the two timing signals transmitted from the detectors to the oscilloscope.

$L=18033.85 \pm 1.25 \mathrm{~mm}$ and $\Delta t_{d}=-99 \pm 4 \mathrm{ps}$ were accurately measured in a dedicated study by using a laser calibration system [24]. Therefore, the task to obtain the ion velocity is reduced to the determination of $\Delta t_{\text {exp }}$ using the measured time sequences.

A straightforward way to deduce $\Delta t_{\exp }$ is to use existing coincident time stamps from both TOF detectors in the same revolution, $\Delta t_{\text {coi }}$. For the ${ }^{45} \mathrm{~V}^{23+}$ ion presented in Fig. 2, 342 $\Delta t_{\text {coi }}$ values, deduced from $\Delta t_{\text {coi }}=t_{\exp }^{\mathrm{TOF} 2}(N)-t_{\exp }^{\mathrm{TOF} 1}(N)$, are presented in Fig. 3 as a function of the revolution number $N$.

The slow increasing trend of $\Delta t_{\text {coi }}$ values with the revolution number in Fig. 3 is due to energy losses of this ion in the thin carbon foils of the TOF detectors. The overall increase of $\Delta t_{\text {coi }}$ from the first to the last revolution is estimated to be $\sim 70 \mathrm{ps}$ which corresponds to $\sim 0.08 \%$ relative variation. The arithmetic mean value of these 342 $\Delta t_{\text {coi }}$ values is $\Delta t_{\exp }=89.738(3) \mathrm{ns}$ and the corresponding

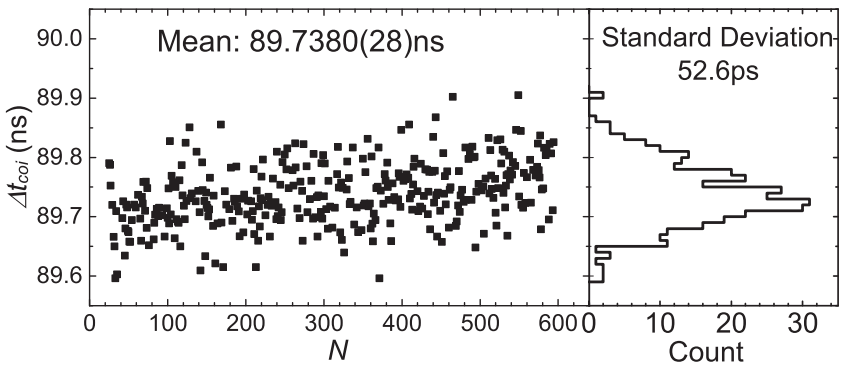

FIG. 3. Time differences, $\Delta t_{\text {coi }}$, as a function of revolution number, $N$, for 342 coincidence timing signals recorded by both TOF detectors in the same revolutions. The data are for the same ${ }^{45} \mathrm{~V}^{23+}$ ion as in Fig. 2. 


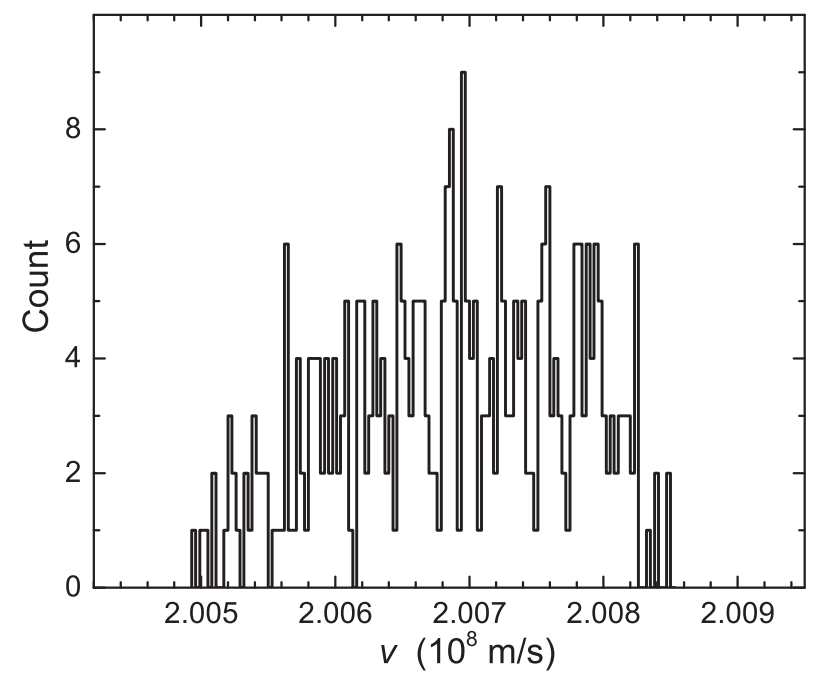

FIG. 4. Velocity distribution of all ${ }^{45} \mathrm{~V}^{23+}$ ions in this experiment extracted from $\Delta t_{\text {coi }}$ values.

mean velocity is determined using Eq. (3) to be $2.00741(6) \times 10^{8} \mathrm{~m} / \mathrm{s}$. Here only statistical errors from $\Delta t_{\text {coi }}$ are included.

Figure 4 shows velocity distribution deduced from Eq. (3) using $\Delta t_{\text {exp }}$ values of all ${ }^{45} \mathrm{~V}^{23+}$ ions detected in this experiment. The obtained range of velocities is $(2.0048-2.0086) \times 10^{8} \mathrm{~m} / \mathrm{s}$, which agrees well with the known acceptance of the CSRe. The velocity values have a typical uncertainty of $6.2 \times 10^{3} \mathrm{~m} / \mathrm{s}$ originated from the statistic errors of $\Delta t_{\text {coi }}$.

From data analyses of mass measurement experiments at the CSRe as well as at the ESR using a single TOF detector, it is well established that the flight time of an ion is a smooth function of the revolution number. If an ion circulates in the ring without energy losses, it would move around a mean orbit of a fixed length. The time sequence of such an ion would thus be described by a linear function with the slope providing directly the mean revolution time. However, due to the inevitable energy losses of the ions passing through the carbon foils of the detectors, the ions slowly change their orbits and the revolution time varies smoothly with the revolution number. Taking this effect into account, a third-order polynomial function is usually used to describe each of the measured time sequences as a function of the revolution number $N[23,40]$. It is defined as

$t_{\mathrm{fit}}(N)=\sum_{i=0}^{3} a_{i} \cdot N^{i}=a_{0}+a_{1} \cdot N+a_{2} \cdot N^{2}+a_{3} \cdot N^{3}$,

where $a_{0}, a_{1}, a_{2}, a_{3}$ are fit parameters. $t_{\text {fit }}(N)$ values are mathematical expectations of the experimental time stamps, $t_{\exp }(N)$, of the ion at the revolution number $N$. Using the fit parameters, the revolution times are derived from the slope of the fitted polynomial function $t_{\text {fit }}(N)[23,40]$. The flight

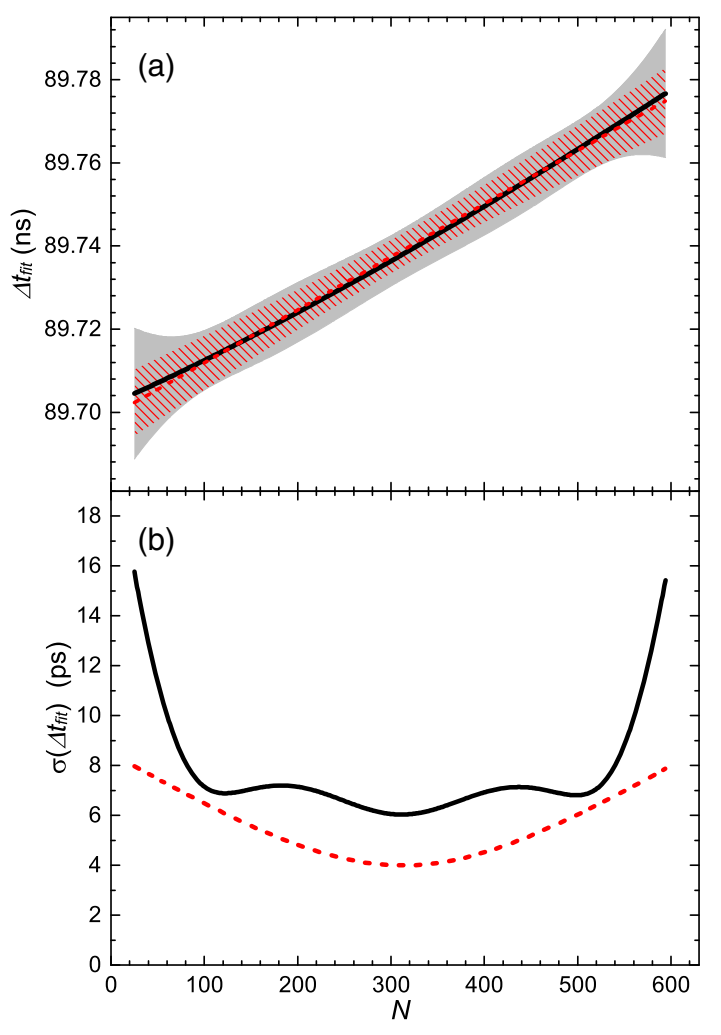

FIG. 5. (a) $\Delta t_{\text {fit }}$ values obtained by using Eq. (5) as a function of the revolution number $N$. The data are for the single ${ }^{45} \mathrm{~V}^{23+}$ ion presented in Fig. 2. (b) The $1 \sigma$ uncertainty of the $\Delta t_{\text {fit }}$ values, $\sigma\left(\Delta t_{\text {fit }}\right)$. The black solid line and gray solid shadow correspond to the fitting with all parameters set free. The red dashed line and red hatched area correspond to the fitting process with $\Delta a_{2}=\Delta a_{3}=0$ (see text).

time between the two TOF detectors, $\Delta t_{\text {fit }}(N)$, can then be determined via

$$
\Delta t_{\mathrm{fit}}(N)=t_{\mathrm{fit}}^{\mathrm{TOF} 2}(N)-t_{\mathrm{fit}}^{\mathrm{TOF} 1}(N)=\sum_{i=0}^{3} \Delta a_{i} \cdot N^{i},
$$

where $\Delta a_{i}=a_{i}^{\mathrm{TOF} 2}-a_{i}^{\mathrm{TOF} 1}(i=0,1,2,3)$. In the experiment, the ions were stored at relativistic energies and the energy losses in the carbon foils were very small with the semi-Landau distribution $[40,41]$. Therefore it can safely be assumed in Eq. (5) that $\Delta a_{2}=\Delta a_{3}=0$ leading to only six free parameters in the fitting procedure.

Figure 5 illustrates the obtained $\Delta t_{\text {fit }}$ values and their uncertainties $\sigma\left(\Delta t_{\text {fit }}\right)$ as a function of the revolution number $N$ for the data of the single ${ }^{45} \mathrm{~V}^{23+}$ ion presented in Fig. 2. The black solid line is the fitted results using Eq. (5) with no additional constraint (eight free parameters). The gray shadowed area shows the corresponding uncertainties of the fitting. The red dashed line and the red hatched area represent the fitted results and the corresponding fit errors with the $\Delta a_{2}=\Delta a_{3}=0$ constraint. Both approaches agree well with each other and the latter one results in smaller 

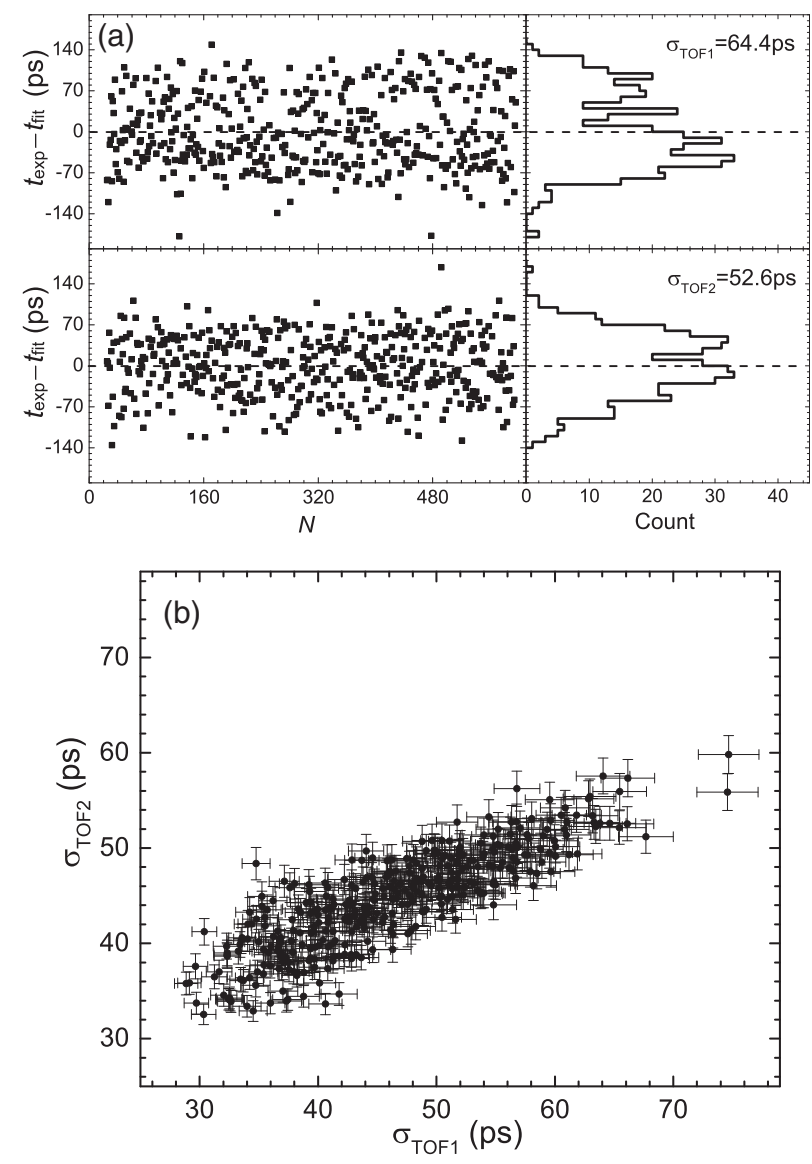

FIG. 6. (a) Residuals of the polynomial fitting as a function of the revolution number $N$ (left) and their projection (right) for the single ${ }^{45} \mathrm{~V}^{23+}$ ion as in Fig. 2. (b) Correlations between $\sigma_{\mathrm{TOF} 2}$ and $\sigma_{\mathrm{TOF} 1}$ for all ${ }^{45} \mathrm{~V}^{23+}$ ions identified in this experiment.

uncertainties. As expected, $\sigma\left(\Delta t_{\text {fit }}\right)$ reaches a minimum at the middle revolution turn [see Fig. 5(b)]. Hereafter and in the subsequent analysis, the condition $\Delta a_{2}=\Delta a_{3}=0$ will be used.

Figure 6(a) depicts the fit residuals of the measured time stamps for the single ${ }^{45} \mathrm{~V}^{23+}$ shown in Fig. 2. The projected histograms of the residuals exhibit broad and obvious nonGaussian distributions. The standard deviations $\sigma_{\mathrm{TOF} 1}=$ $64.4 \mathrm{ps}$ and $\sigma_{\mathrm{TOF} 2}=52.6 \mathrm{ps}$ are larger than the measured intrinsic time resolutions of the detectors of $\sim 30 \mathrm{ps}$ $[21,24]$. Furthermore, they are larger than, or accidentally equal to, the $\sigma\left(\Delta t_{\mathrm{coi}}\right)$ value shown in Fig. 3.

Figure 6(b) illustrates the scattering plot of the extracted $\sigma_{\mathrm{TOF} 2}$ versus $\sigma_{\mathrm{TOF} 1}$ for all ${ }^{45} \mathrm{~V}^{23}+$ ions detected in this experiment. A clear correlation is observed between $\sigma_{\mathrm{TOF} 1}$ and $\sigma_{\mathrm{TOF} 2}$ with a fairly large Pearson correlation coefficient of 0.85 . Such correlations are also observed for all other ion species. The double-peak structures of fit residuals from both detectors in Fig. 6(a) and the high correlation between $\sigma_{\mathrm{TOF} 1}$ and $\sigma_{\mathrm{TOF} 2}$ in Fig. 6 (b) point to an unconsidered systematic effect that requires further treatment.

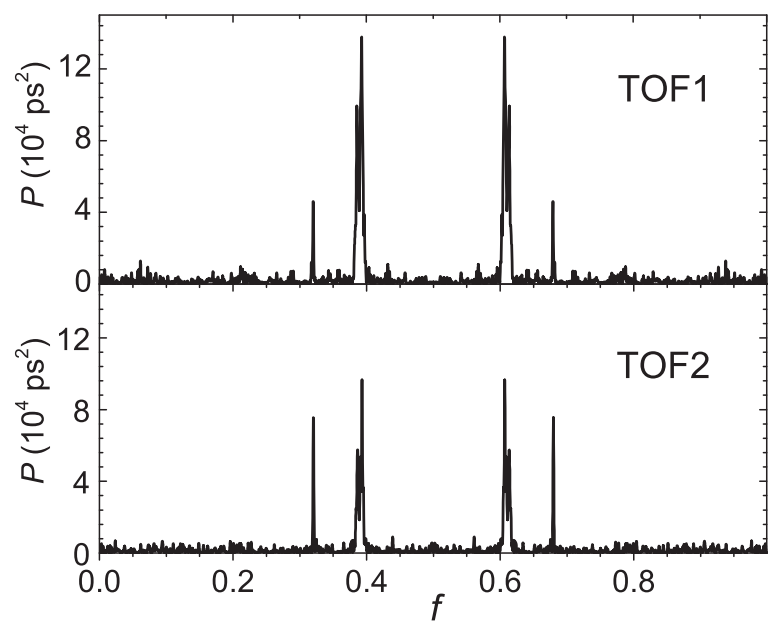

FIG. 7. Periodogram extracted from the fit residuals in Fig. 6(a).

It is well known that the motion of a charged particle with a nonzero emittance in a storage ring is characterized by betatron oscillations in the transverse plane of the ion motion [33]. This may cause extra periodic fluctuations in the orbital length and thus the revolution time will oscillate as a function of the revolution number [28]. To check possible influences of these emittance-induced oscillations, each of the fit residuals shown in Fig. 6(a) has been analyzed with the discrete Fourier transform technique. A periodogram, $P(f)$, conventionally defined as [42]

$$
\begin{aligned}
P(f)= & \frac{1}{n_{s}}\left\{\left[\sum_{j}^{n_{s}} X_{N_{j}} \cdot \cos \left(2 \pi f \cdot N_{j}\right)\right]^{2}\right. \\
& \left.+\left[\sum_{j}^{n_{s}} X_{N_{j}} \cdot \sin \left(2 \pi f \cdot N_{j}\right)\right]^{2}\right\},
\end{aligned}
$$

was applied for the detection of periodic components hidden in the residuals. Here $X_{N_{j}}$ is the fit residual at the revolution number $N_{j}$ and $n_{s}$ is the total number of time stamps. The presence of a periodic component in the residuals shall be indicated by a distinct narrow peak in the $P(f)$ spectrum [42].

The periodogram analysis yielded the $P(f)$ spectrum shown in Fig. 7, which is symmetrical against $f=0.5$. Two predominant peaks are found in the $0 \leq f \leq 0.5$ range at $f \approx 0.32$ and 0.39 for both TOF1 and TOF2 time sequences. By doing the same analysis for other time sequences of all detected ions, we find that the peaks are always at nearly the same $f$ values, but with different amplitudes. This result indicates that the peak positions in the $P(f)$ spectrum are determined by the machine properties of the storage ring, most likely the tune values of betatron oscillations. In the horizontal and vertical directions in the transverse plane of the ion motion, the tunes are defined as the numbers of betatron oscillations per revolution, denoted by $Q_{x}$ and $Q_{y}$ 
respectively [34]. In the next section it will be shown that the two frequencies indicated by the peaks near 0.39 and 0.32 in Fig. 7 are the fraction parts of $Q_{x}$ and $Q_{y}$, respectively.

To account for betatron oscillations, the fit function of Eq. (4) was modified by adding two additional sinelike terms:

$$
\begin{aligned}
t_{\mathrm{fit}}(N)= & \sum_{i=0}^{3} a_{i} \cdot N^{i}+A_{x} \cdot \sin \left[2 \pi \cdot\left(Q_{x 0} \cdot N+Q_{x 1} \cdot N^{2}\right)+\phi_{x}\right] \\
& +A_{y} \cdot \sin \left[2 \pi \cdot\left(Q_{y 0} \cdot N+Q_{y 1} \cdot N^{2}\right)+\phi_{y}\right] .
\end{aligned}
$$

The polynomial function of Eq. (7) describes in fact the ion motion with a mean orbital length, while the sinelike terms describe the periodic time fluctuations due to betatron oscillations. The first derivative of the phase term in Eq. (7), $Q=Q_{0}+2 Q_{1} \cdot N$, represents the fractional horizontal/ vertical tune. The tune variation factors, $Q_{x 1}$ and $Q_{y 1}$, which are the results of the ion energy loss in the TOF foils, are proportional to the corresponding chromaticities of the ring [34]. In the fitting procedure, the constraints
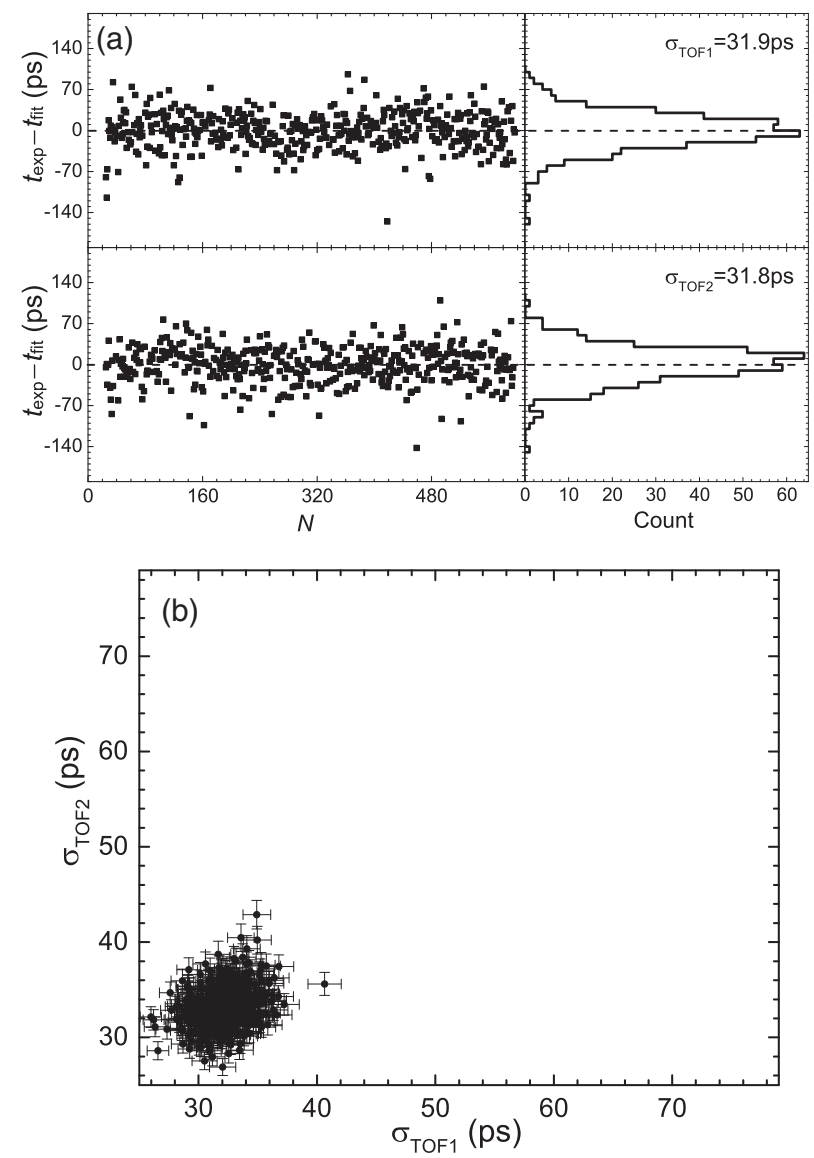

FIG. 8. Same as Fig. 6, but using Eq. (7) in the fitting procedure.

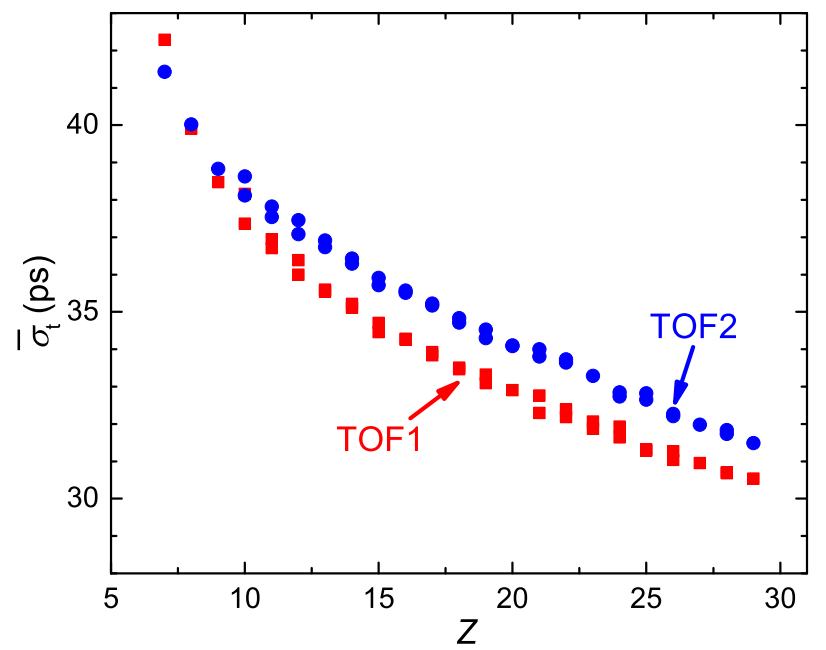

FIG. 9. Z dependence of the experimental time resolution of the TOF detectors deduced from the fit residuals using Eq. (7).

$Q_{0}^{\mathrm{TOF} 1}=Q_{0}^{\mathrm{TOF} 2}$ and $Q_{1}^{\mathrm{TOF} 1}=Q_{1}^{\mathrm{TOF} 2}$ were used since the two TOF detectors are measuring the same ion.

The results using the updated fit function of Eq. (7) are shown in Fig. 8. The standard deviations of the fit residuals are reduced to $\sigma_{\mathrm{TOF} 1}=31.9 \mathrm{ps}$ and $\sigma_{\mathrm{TOF} 2}=31.8 \mathrm{ps}$, which are now closer to the intrinsic time resolutions of $\sim 30 \mathrm{ps}$ of the TOF detectors [21]. The correlation between $\sigma_{\mathrm{TOF} 2}$ and $\sigma_{\mathrm{TOF} 1}$ observed in Fig. $6(\mathrm{~b})$ has been removed as shown in Fig. 8(b). Both results in Fig. 8 indicate that the time fluctuations induced by the betatron oscillations have been-at least partly-described by Eq. (7).

It is clear that the new fitted parameters, $a_{i}(i=0,1,2,3)$, using Eq. (7) are different from those when using Eq. (4). The new parameters are used in Eq. (5) to deduce the flight time, $\Delta t_{\text {fit }}(N)$, between the two TOF detectors. Taking $\Delta t_{\text {exp }}=$ $\Delta t_{\text {fit }}(N)$ at the middle revolution number, the velocity of the ${ }^{45} \mathrm{~V}^{23+}$ ion presented in Fig. 2 is determined to be $2.00735(5) \times 10^{8} \mathrm{~m} / \mathrm{s}$ via Eq. (3) which is in good agreement with the result obtained by using the $\Delta t_{\text {coi }}$ values.

Figure 9 shows $\mathrm{Z}$ dependence of the experimental time resolution of the TOF detectors deduced from the fit residuals using Eq. (7). The number of secondary electrons released from the foil of the TOF detector is smaller for lower-Z ions. This affects the detection efficiency as well as accuracy of measured timing signals and, as a consequence, statistical uncertainties for lighter-Z ions are larger.

\section{RESULTS AND DISCUSSION}

\section{A. Velocity}

Precision velocity measurement of a stored ion depends critically on the precision determination of its time of flight, $\Delta t_{\text {exp }}$, between the two TOF detectors. It turned out that at the present level of accuracies, it became essential to include two sinelike terms into the fit function to describe the betatron oscillations of the ion motion in the storage 


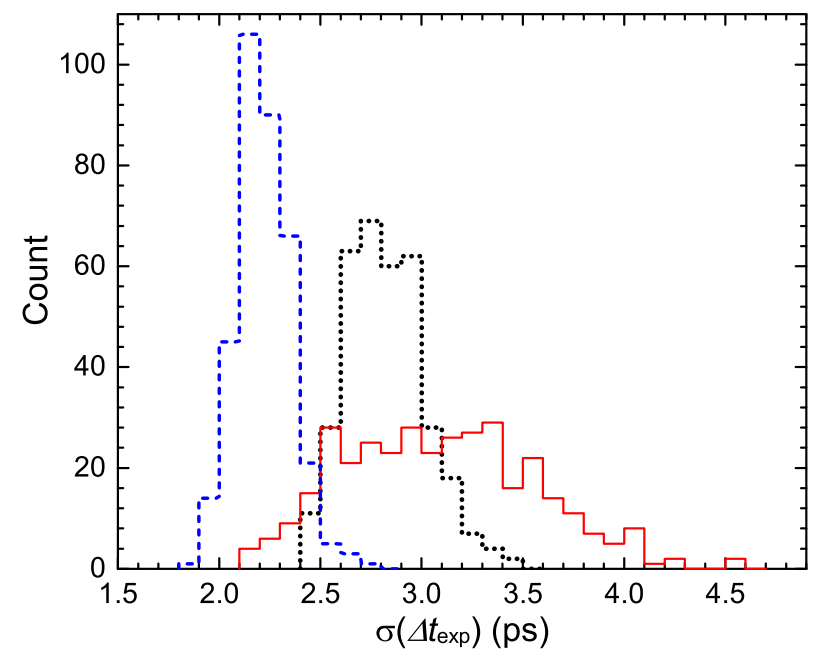

FIG. 10. Uncertainty distributions of $\Delta t_{\exp }$ for all ${ }^{45} \mathrm{~V}^{23+}$ ions extracted by averaging the time differences, $\Delta t_{\text {coi }}$, of the coincident time stamps in different revolutions (black dotted line), and by using the polynomial fit function with [Eq. (7)] (blue dashed line) and without [Eq. (4)] (red solid line) the sinelike terms.

ring. Figure 10 illustrates time uncertainties, $\sigma\left(\Delta t_{\text {exp }}\right)$, of $\Delta t_{\text {exp }}$ values for all stored ${ }^{45} \mathrm{~V}^{23}+$ ions determined via three approaches, namely (i) by averaging the individual times of flight, $\Delta t_{\text {coi }}$, deduced from the coincident time stamps in different revolutions, (ii) by a polynomial fit function according to Eq. (4), and (iii) by a polynomial fit function with two sinelike terms included according to Eq. (7). One can clearly see that the smallest mean value of $\sigma\left(\Delta t_{\text {exp }}\right)$ has been obtained in the latter case. This indicates that properly considering and treating the effects of the betatron oscillations can significantly improve the precision of the $\Delta t_{\exp }$ values and thus the velocities of the ions.

The analysis described above has been applied to all ion species with more than 100 ions detected, yielding similar distributions as in Fig. 10. The mean time uncertainties, $\bar{\sigma}\left(\Delta t_{\exp }\right)$, for each ion species have been extracted and plotted in Fig. 11. This figure shows that the mean uncertainties have a decreasing trend from lighter to heavier ions. This can be understood if the $Z$ dependence of the detection efficiency of the detectors is considered, i.e., a higher detection efficiency gives lower statistical errors in $\Delta t_{\text {exp }}$.

The lowest mean uncertainties of $\Delta t_{\exp }$ are within the range of 2.0-6.4 ps, corresponding to the relative precision of $(2.2-7.2) \times 10^{-5}$ with respect to an average time of flight between the two TOF detectors of $\Delta t_{\exp } \approx 89 \mathrm{~ns}$. At present, the best single-path time resolution in the TOF mass spectrometry has been achieved to be less than $10 \mathrm{ps}$ by using fast plastic scintillators [43]. In our IMS experiments in the CSRe, although the intrinsic time resolutions

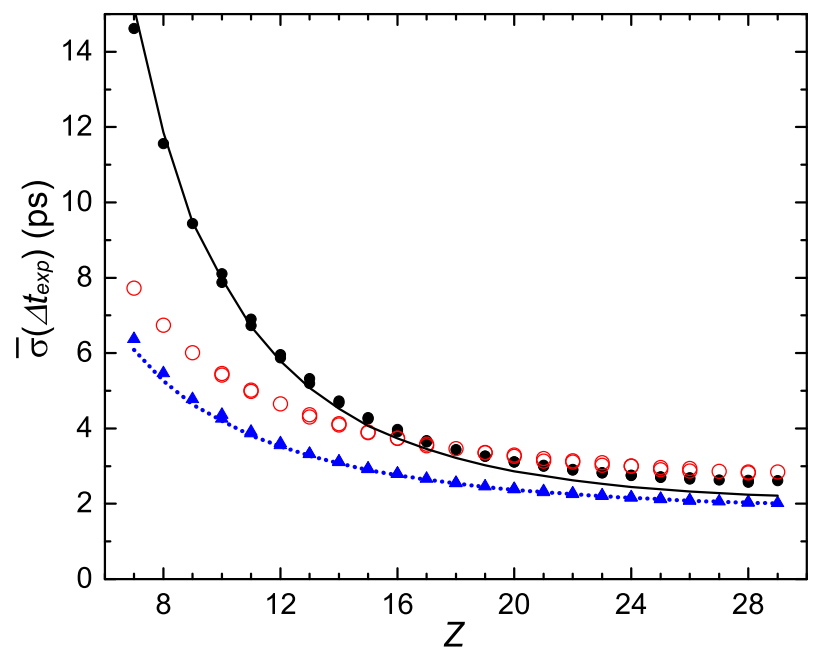

FIG. 11. Average uncertainty of $\Delta t_{\exp }$ as a function of atomic number (Z), extracted from available $\Delta t_{\text {coi }}$ values (black filled circles), and by using the third-order polynomial fit function with [Eq. (7)] (blue filled triangles) and without [Eq. (4)] (red opened circles) the sinelike terms. Solid and dotted lines represent the estimations using Eqs. (8) and (9), respectively.

of TOF detectors are about $30 \mathrm{ps,} \mathrm{such} \mathrm{high} \mathrm{precision} \mathrm{is}$ achieved thanks to the in-ring multiturn measurements.

To understand the mean uncertainties, $\bar{\sigma}\left(\Delta t_{\text {exp }}\right)$, and their variation trends shown in Fig. 11, an error estimation can be made. The expected uncertainty of $\Delta t_{\exp }$ deduced from one-to-one coincident time stamps is given by

$$
\sigma\left(\Delta t_{\exp }\right)=\sqrt{\frac{\sigma_{1}^{2}+\sigma_{2}^{2}}{N_{t} \times \eta_{1} \times \eta_{2}}},
$$

where $\sigma_{i}(i=1,2)$ and $\eta_{i}(i=1,2)$ stand for the time resolutions and detection efficiencies of the detectors, respectively, and $N_{t}$ is the total number of revolutions. The mean detection efficiencies, $\eta_{1}$ and $\eta_{2}$, and the number of revolutions, $N_{t}$, for the specific ion species were deduced from the observed time sequences. By using the experimental time resolutions shown in Fig. 9, the experimental $\bar{\sigma}\left(\Delta t_{\exp }\right)$ and its $Z$ dependence can be fairly well reproduced with Eq. (8) (see solid line in Fig. 11). The experimental $\bar{\sigma}\left(\Delta t_{\exp }\right)$ values are slightly larger than the estimations for the higher- $Z$ nuclei, this could be due to the effects of energy losses as shown in Fig. 3.

If $\Delta t_{\exp }$ values are deduced through the fitting procedure by using all time stamps, the $\bar{\sigma}\left(\Delta t_{\exp }\right)$ can be estimated by a modified formula:

$$
\sigma\left(\Delta t_{\exp }\right)=\sqrt{\frac{\sigma_{1}^{2}}{N_{t} \times \eta_{1}}+\frac{\sigma_{2}^{2}}{N_{t} \times \eta_{2}}} .
$$


The estimated errors, $\sigma\left(\Delta t_{\text {exp }}\right)$, are plotted with a dotted line in Fig. 11. The estimated results are in perfect agreement with the values obtained experimentally.

\section{B. $Q$ values and the chromaticity of the CSRe}

As mentioned above, it is likely that the additional sinelike terms in Eq. (7) describe the periodic time fluctuations originated from the betatron oscillations of the stored ions. On the one hand, when an ion with the emittances $\varepsilon_{x, y}$ moves in the ring with betatron tunes $Q_{x, y}$, its orbit length will oscillate around the mean orbit length, leading to the oscillation of the flight times in the ring. The amplitude of such orbit-length oscillation in the bending plane, $\Delta C_{x}$, can be calculated by [34]

$$
\begin{aligned}
\Delta C_{x} & =\frac{\sqrt{\varepsilon_{x}}}{2 \sin \left(Q_{x} \pi\right)} \sqrt{\gamma_{x} R_{52}{ }^{2}-2 \alpha_{x} R_{52} R_{51}+\beta_{x} R_{51}{ }^{2}} \\
& =\frac{\sqrt{\varepsilon_{x}}}{2 \sin \left(Q_{x} \pi\right)} \sqrt{\gamma_{x} R_{16}{ }^{2}+2 \alpha_{x} R_{16} R_{26}+\beta_{x} R_{26}{ }^{2}} \\
& =\sqrt{\varepsilon_{x}} \sqrt{\gamma_{x} D_{x}{ }^{2}+2 \alpha_{x} D_{x} D_{x}^{\prime}+\beta_{x} D_{x}^{\prime 2}},
\end{aligned}
$$

where $\alpha_{x}, \beta_{x}$, and $\gamma_{x}$ are the Courant-Snyder or Twiss parameters at the TOF position, $R_{51}, R_{52}, R_{16}$, and $R_{26}$ are the elements of the one-turn transport matrix from and to the TOF detector, and $D_{x}$ and $D_{x}^{\prime}$ are the dispersion functions at the detector position. Since the dispersions $D_{y}$ and $D_{y}^{\prime}$ are typically equal to zero, as shown in Fig. 1, the particle emittance in the vertical plane, $\varepsilon_{y}$, should not contribute to the orbital-length oscillation (equivalently to the flight-time oscillation) if not considering the coupling of betatron oscillations in $\hat{x}$ and $\hat{y}$ directions.

On the other hand, the ion circulating with a betatron oscillation would hit the carbon foil of the TOF detector at different positions during its revolutions in the ring. This would result in the hit-position oscillations in both $\hat{x}$ and $\hat{y}$ directions with two frequencies, namely $Q_{x}$ and $Q_{y}$, respectively. The amplitudes of such position oscillations are determined by $\sqrt{\varepsilon_{x, y} \cdot \beta_{x, y}}$. The ion optics of the real TOF detector is not perfectly isochronous due to imperfections of the electric and magnetic fields, which are employed in the detector to guide secondary electrons from the carbon foil to a microchannel plate. Hence, the transmission times of the secondary electrons in the detector are likely to be position dependent [21]. This can as well contribute to the observed flight-time oscillations.

Taking into account the oscillations of both the orbit lengths and the hit positions along with the intrinsic nonisochronicity of the TOF detector, it is evident that the emittance, $\varepsilon_{x}$, of the stored ion induces the flight-time oscillation with the frequency $Q_{x}$. The hit-position oscillations in the $\hat{y}$ direction with the frequency $Q_{y}$ might be coupled via the not perfectly isochronous TOF detector and imposed onto the oscillations of the measured flight times,

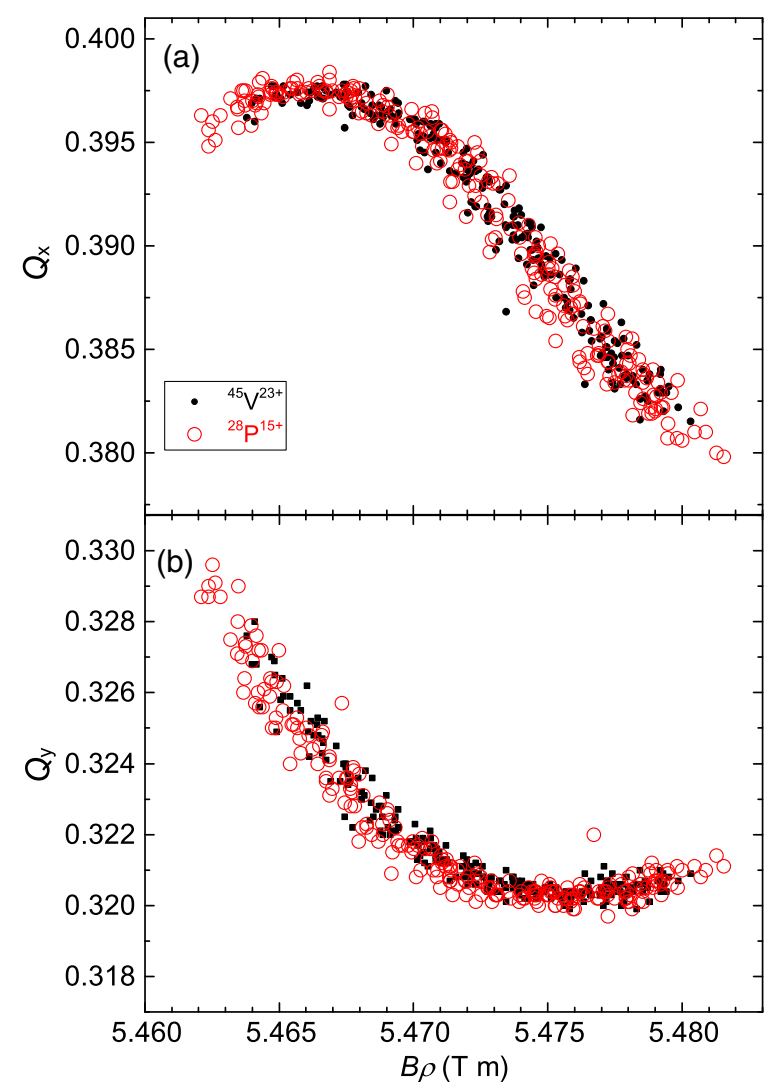

FIG. 12. Scattering plot of fitted $Q=Q_{0}+2 Q_{1} \cdot N$ values at the middle revolution number, $Q_{x}$ (a) and $Q_{y}(\mathrm{~b})$, for ${ }^{45} \mathrm{~V}^{23+}$ (black dot) and ${ }^{28} \mathrm{P}^{15+}$ (red circle) ions. The magnetic rigidities, $B \rho$, of the ions are calculated according to the known masses [44] and the measured velocities. Only the ions with a condition of $A^{\mathrm{TOF} 2}>$ 15 ps in Eq. (7) are used corresponding to $68 \%$ of the whole events.

although $\varepsilon_{y}$ should not directly contribute to the orbitallength oscillation $\Delta C$. The fitting procedure using Eq. (7) can yield the two tune values $Q_{x}$ and $Q_{y}$, but it is not possible to separate the contributions from the betatron oscillation of the ion in the ring lattice and from the transmission times of the electrons in the TOF detector.

Mathematically, it makes no difference to use the peak frequency $f$ or $1-f$ shown in Fig. 7 in the fitting procedure with Eq. (7). Because $Q_{x} \approx Q_{y} \approx 2.37$ in the lattice design in this experiment, the measured two frequencies below 0.5 are assigned to be the fraction parts of the tune values. It is also suggested to assign the peak $f \approx 0.39$ to be associated with $Q_{x}$ as it has larger amplitude than the other one.

The fitted parameters, $Q=Q_{0}+2 Q_{1} \cdot N$ at the middle revolution number for ${ }^{45} \mathrm{~V}^{23+}$ and ${ }^{28} \mathrm{P}^{15+}$ ions are shown in Fig. 12. The magnetic rigidities, $B \rho$, of the ions are calculated according to the known masses [44] and the measured velocities. As expected, the fitted $Q$ values are independent of the ion species as shown in Fig. 12. The fitted $Q$ values vary with the magnetic rigidities of the 
stored ions, thus reflecting the chromaticity of the CSRe. The tunes and the chromaticities will be measured in future experiments with an independent method to cross-check the results presented here.

\section{CONCLUSION}

To realize a novel scheme of IMS, two TOF detectors were installed in a straight section of the CSRe in Lanzhou. Revolution times as well as velocities of the stored ions have been measured by using this new experimental setup. The analysis of the first data revealed that the measured time sequences from ions passing the TOF detectors are sensitive to betatron oscillations of the ion motion in the ring. Two sinelike terms were added to describe the flight time oscillations of the ions as a function of the revolution number. The relative precisions at a level of $\bar{\sigma}\left(\Delta t_{\exp }\right) / \Delta t_{\exp } \sim 10^{-5}$ have been achieved. The betatron tunes of the CSRe were obtained with high precision for the first time by the novel data analysis method developed in this work. With the successful precision in-ring velocity measurements of each stored ion, a new way to study the chromaticities of the storage ring has been developed. The merit of this new way is the sensitivity that the measurements can be conducted using a secondary beam with only several or even a single stored ion in the storage ring.

At the future HIAF facility [45,46], two TOF detectors equipped with ultrathin carbon foils of even larger diameters will be installed in one of the straight sections of the spectrometer ring (SRing) [47]. The distance between the detectors will be longer than in the case of the CSRe. The same concept will be followed in the ILIMA project at the FAIR facility in Germany [30,48,49], where two TOF detectors will be installed in the collector ring (CR) $[25,50]$. The results obtained and techniques developed in this work can be essential for designing the experiments at these future storage rings.

\section{ACKNOWLEDGMENTS}

This work is supported by the National Key R\&D Program of China (Grants No. 2016YFA0400504 and No. 2018YFA0404401), the Strategic Priority Research Program of Chinese Academy of Sciences (Grant No. XDB34000000), and the NSFC (Grants No. 11905261, No. 11805032, No. 11975280, and No. 11605248). Y.M.X. is thankful for support from the CAS "Light of West China" Program, X. X. acknowledges support from China Postdoctoral Science Foundation (Contract No. 2019M660250), X. Y. acknowledges partial support by the FRIB-CSC Fellowship, China, under Grant No. 201704910964, R. J. C. is supported by the International Postdoctoral Exchange Fellowship Program 2017 by the Office of China Postdoctoral Council (No. 60 Document of OCPC, 2017), and Y.A.L. and R.S.S. received support from the European Research Council
(ERC) under the European Union's Horizon 2020 research and innovation program (Grant Agreement No. 682841 "ASTRUm"). Japanese authors were supported in part by JSPS and NSFC under the "Japan-China Scientific Cooperation Program".

[1] K. Blaum, High-accuracy mass spectrometry with stored ions, Phys. Rep. 425, 1 (2006).

[2] Y. H. Zhang, Y. A. Litvinov, T. Uesaka, and H. S. Xu, Storage ring mass spectrometry for nuclear structure and astrophysics research, Phys. Scr. 91, 073002 (2016).

[3] M. Steck and Y. Litvinov, Heavy-ion storage rings and their use in precision experiments with highly charged ions, Prog. Part. Nucl. Phys. 115, 103811 (2020).

[4] M. Hausmann, F. Attallah, K. Beckert, F. Bosch, A. Dolinskiy, H. Eickhoff, M. Falch, B. Franczak, B. Franzke, H. Geissel et al., First isochronous mass spectrometry at the experimental storage ring ESR, Nucl. Instrum. Methods Phys. Res., Sect. A 446, 569 (2000).

[5] J. Stadlmann, M. Hausmann, F. Attallah, K. Beckert, P. Beller, F. Bosch, H. Eickhoff, M. Falch, B. Franczak, B. Franzke et al., Direct mass measurement of bare short-lived ${ }^{44} \mathrm{~V},{ }^{48} \mathrm{Mn},{ }^{41} \mathrm{Ti}$ and ${ }^{45} \mathrm{Cr}$ ions with isochronous mass spectrometry, Phys. Lett. B 586, 27 (2004).

[6] H. S. Xu, Y. H. Zhang, and Y. A. Litvinov, Accurate mass measurements of exotic nuclei with the CSRe in Lanzhou, Int. J. Mass Spectrom. 349-350, 162 (2013).

[7] M. Wang, H. S. Xu, J. W. Xia, X. L. Tu, R. S. Mao, Y. J. Yuan, Z. G. Hu, Y. Liu, H. B. Zhang, Y. D. Zang et al., First isochronous mass measurements at CSRe, Int. J. Mod. Phys. E 18, 352 (2009).

[8] T. Yamaguchi, Y. Yamaguchi, and A. Ozawa, The challenge of precision mass measurements of short-lived exotic nuclei: Development of a new storage ring mass spectrometry, Int. J. Mass Spectrom. 349-350, 240 (2013).

[9] Y. Yamaguchi, M. Wakasugi, T. Uesaka, A. Ozawa, Y. Abe, T. Fujinawa, M. Kase, M. Komiyama, T. Kubo, K. Kumagai et al., Construction of rare-RI ring at RIKEN RI Beam Factory, Nucl. Instrum. Methods Phys. Res., Sect. B 317, 629 (2013).

[10] X. Xu, M. Wang, K. Blaum, J. D. Holt, Y. A. Litvinov, A. Schwenk, J. Simonis, S. R. Stroberg, Y. H. Zhang, H. S. Xu et al., Masses of neutron-rich ${ }^{52-54} \mathrm{Sc}$ and ${ }^{54,56} \mathrm{Ti}$ nuclides: The $n=32$ subshell closure in scandium, Phys. Rev. C 99, 064303 (2019).

[11] X. Xu, J. H. Liu, C. X. Yuan, Y. M. Xing, M. Wang, Y. H. Zhang, X. H. Zhou, Y. A. Litvinov, K. Blaum, R. J. Chen et al., Masses of ground and isomeric states of ${ }^{101} \mathrm{In}$ and configuration-dependent shell evolution in odd- $a$ indium isotopes, Phys. Rev. C 100, 051303(R) (2019).

[12] Y. H. Zhang, P. Zhang, X. H. Zhou, M. Wang, Y. A. Litvinov, H. S. Xu, X. Xu, P. Shuai, Y. H. Lam, R. J. Chen et al., Isochronous mass measurements of $T_{z}=-1 \mathrm{fp}$ shell nuclei from projectile fragmentation of ${ }^{58} \mathrm{Ni}$, Phys. Rev. C 98, 014319 (2018).

[13] C. Y. Fu, Y. H. Zhang, X. H. Zhou, M. Wang, Y. A. Litvinov, K. Blaum, H. S. Xu, X. Xu, P. Shuai, Y. H. Lam et al., Masses 
of the $T_{z}=-3 / 2$ nuclei ${ }^{27} \mathrm{P}$ and ${ }^{29} \mathrm{~S}$, Phys. Rev. C 98, 014315 (2018).

[14] Y. M. Xing, K. A. Li, Y. H. Zhang, X. H. Zhou, M. Wang, Y. A. Litvinov, K. Blaum, S. Wanajo, S. Kubono, G. Martínez-Pinedo et al., Mass measurements of neutrondeficient $\mathrm{Y}, \mathrm{Zr}$, and $\mathrm{Nb}$ isotopes and their impact on $r p$ and $\nu p$ nucleosynthesis processes, Phys. Lett. B 781, 358 (2018).

[15] Q. Zeng, M. Wang, X. H. Zhou, Y. H. Zhang, X. L. Tu, X. C. Chen, X. Xu, Y. A. Litvinov, H. S. Xu, K. Blaum et al., Half-life measurement of short-lived ${ }_{44}^{94 m} \mathrm{ru}^{44+}$ using isochronous mass spectrometry, Phys. Rev. C 96, 031303 (R) (2017).

[16] P. Zhang, X. Xu, P. Shuai, R. J. Chen, X. L. Yan, Y. H. Zhang, M. Wang, Y. A. Litvinov, K. Blaum, H. S. Xu et al., High-precision $Q_{\mathrm{EC}}$ values of superallowed $0^{+} \rightarrow 0^{+} \beta$ emitters ${ }^{46} \mathrm{Cr},{ }^{50} \mathrm{Fe}$ and ${ }^{54} \mathrm{Ni}$, Phys. Lett. B 767, 20 (2017).

[17] X. Xu, P. Zhang, P. Shuai, R. J. Chen, X. L. Yan, Y. H. Zhang, M. Wang, Y. A. Litvinov, H. S. Xu, T. Bao et al., Identification of the Lowest $t=2, J^{\pi}=0^{+}$Isobaric Analog State in ${ }^{52} \mathrm{Co}$ and Its Impact on the Understanding of $\beta$-Decay Properties of ${ }^{52} \mathrm{Ni}$, Phys. Rev. Lett. 117, 182503 (2016).

[18] X. L. Tu, H. S. Xu, M. Wang, Y. H. Zhang, Y. A. Litvinov, Y. Sun, H. Schatz, X. H. Zhou, Y. J. Yuan, J. W. Xia et al., Direct Mass Measurements of Short-Lived $A=2 Z-1$ Nuclides ${ }^{63} \mathrm{Ge},{ }^{65} \mathrm{As},{ }^{67} \mathrm{Se}$, and ${ }^{71} \mathrm{Kr}$ and their Impact on Nucleosynthesis in the $r p$ Process, Phys. Rev. Lett. 106, 112501 (2011).

[19] X. L. Yan, H. S. Xu, Y. A. Litvinov, Y.H. Zhang, H. Schatz, X. L. Tu, K. Blaum, X. H. Zhou, B. H. Sun, J. J. He et al., Mass measurement of ${ }^{45} \mathrm{Cr}$ and its impact on the Ca-Sc cycle in x-ray bursts, Astrophys. J. 766, L8 (2013).

[20] B. Mei, X. L. Tu, M. Wang, H. S. Xu, R. S. Mao, Z. G. Hu, X. W. Ma, Y. J. Yuan, X. Y. Zhang, P. Geng et al., A high performance time-of-flight detector applied to isochronous mass measurement at CSRe, Nucl. Instrum. Methods Phys. Res., Sect. A 624, 109 (2010).

[21] W. Zhang, X. L. Tu, M. Wang, Y. H. Zhang, H. S. Xu, Y. A. Litvinov, K. Blaum, R. J. Chen, X. C. Chen, C. Y. Fu et al., Time-of-flight detectors with improved timing performance for isochronous mass measurements at the CSRe, Nucl. Instrum. Methods Phys. Res., Sect. A 756, 1 (2014).

[22] B. Franzke, H. Geissel, and G. Münzenberg, Mass and lifetime measurements of exotic nuclei in storage rings, Mass Spectrom. Rev. 27, 428 (2008).

[23] X. L. Tu, M. Wang, Y. A. Litvinov, Y. H. Zhang, H. S. Xu, Z. Y. Sun, G. Audi, K. Blaum, C. M. Du, W.X. Huang et al., Precision isochronous mass measurements at the storage ring CSRe in Lanzhou, Nucl. Instrum. Methods Phys. Res., Sect. A 654, 213 (2011).

[24] X. L. Yan, R. J. Chen, M. Wang, Y. J. Yuan, J. D. Yuan, S. M. Wang, G. Z. Cai, M. Zhang, Z. W. Lu, C. Y. Fu et al., Characterization of a double time-of-flight detector system for accurate velocity measurement in a storage ring using laser beams, Nucl. Instrum. Methods Phys. Res., Sect. A 931, 52 (2019).

[25] A. Dolinskii, H. Geissel, S. Litvinov, F. Nolden, M. Steck, and $\mathrm{H}$. Weick, The CR storage ring in an isochronous mode operation with nonlinear optics characteristics, Nucl. Instrum. Methods Phys. Res., Sect. B 266, 4579 (2008).

[26] J. W. Xia, W. L. Zhan, B. W. Wei, Y. J. Yuan, M. T. Song, W. Z. Zhang, X. D. Yang, P. Yuan, D. Q. Gao, H. W. Zhao et al., The heavy ion cooler-storage-ring project (HIRFLCSR) at Lanzhou, Nucl. Instrum. Methods Phys. Res., Sect. A 488, 11 (2002).

[27] W. W. Ge, Y. J. Yuan, J. C. Yang, R. J. Chen, X. L. Yan, H. Du, Z. S. Li, J. Yang, D. Y. Yin, L. J. Mao et al., Experimental investigation of the transition energy $\gamma_{t}$ in the isochronous mode of the HIRFL-CSRe, Nucl. Instrum. Methods Phys. Res., Sect. A 908, 388 (2018).

[28] R. J. Chen, Y. J. Yuan, M. Wang, X. Xu, P. Shuai, Y. H. Zhang, X. L. Yan, Y. M. Xing, H. S. Xu, X. H. Zhou et al., Simulations of the isochronous mass spectrometry at the HIRFL-CSR, Phys. Scr. T166, 014044 (2015).

[29] H. Geissel and Y. A. Litvinov, Precision experiments with relativistic exotic nuclei at GSI, J. Phys. G 31, S1779 (2005).

[30] P. M. Walker et al., ILIMA Technical Proposal, GSI, 2005.

[31] H. Geissel, R. Knöbel, Y. A. Litvinov, B. Sun, K. Beckert, P. Beller, F. Bosch, D. Boutin, C. Brandau, L. Chen et al., A new experimental approach for isochronous mass measurements of short-lived exotic nuclei with the frsesr facility, Hyperfine Interact. 173, 49 (2006).

[32] J. H. Liu, X. Xu, P. Zhang, P. Shuai, X. L. Yan, Y. H. Zhang, M. Wang, Y. A. Litvinov, H. S. Xu, K. Blaum et al., Improving the resolving power of isochronous mass spectrometry by employing an in-ring mechanical slit, Nucl. Instrum. Methods Phys. Res., Sect. B 463, 138 (2020).

[33] E. D. Courant and H. S. Snyder, Theory of the alternatinggradient synchrotron, Ann. Phys. (N.Y.) 3, 360 (1958).

[34] S. Y. Lee, Accelerator Physics (Second Edition) (World Scientific Publishing Co. Pte. Ltd., Singapore, 2004).

[35] P. Bryant, AGILE, A tool for interactive lattice design, in Proceedings of the European Particle Accelerator Conference, Vienna, 2000 (EPS, Geneva, 2000).

[36] X. Xu, M. Wang, P. Shuai, R. J. Chen, X. L. Yan, Y. H. Zhang, Y. J. Yuan, H. S. Xu, X. H. Zhou, Y. A. Litvinov et al., A data analysis method for isochronous mass spectrometry using two time-of-flight detectors at csre, Chin. Phys. C 39, 106201 (2015).

[37] Y. M. Xing, M. Wang, Y. H. Zhang, P. Shuai, X. Xu, R. J. Chen, X. L. Yan, X. L. Tu, W. Zhang, C. Y. Fu et al., First isochronous mass measurements with two time-of-flight detectors at CSRe, Phys. Scr. T166, 014010 (2015).

[38] P. Shuai, X. Xu, Y. H. Zhang, H. S. Xu, Y. A. Litvinov, M. Wang, X. L. Tu, K. Blaum, X. H. Zhou, Y. J. Yuan et al., An improvement of isochronous mass spectrometry: Velocity measurements using two time-of-flight detectors, Nucl. Instrum. Methods Phys. Res., Sect. B 376, 311 (2016).

[39] Y. Xing, Y. Zhang, M. Wang, Y. Litvinov, R. Chen, X. Chen, C. Fu, H. Li, P. Shuai, M. Si et al., Particle identification and revolution time corrections for the isochronous mass spectrometry in storage rings, Nucl. Instrum. Methods Phys. Res., Sect. A 941, 162331 (2019).

[40] R. J. Chen, X. L. Yan, W. W. Ge, Y. J. Yuan, M. Wang, M. Z. Sun, Y. M. Xing, P. Zhang, C. Y. Fu, P. Shuai et al., 
A method to measure the transition energy $\gamma_{t}$ of the isochronously tuned storage ring, Nucl. Instrum. Methods Phys. Res., Sect. A 898, 111 (2018).

[41] P. A. Zyla, R. M. Barnett, J. Beringer, O. Dahl, D. A. Dwyer, D. E. Groom, C. J. Lin, K. S. Lugovsky, E. Pianori, D. J. Robinson et al., Review of particle physics, Rev. Part. Phys. 2020, 083C01 (2020).

[42] J. D. Scargle, Studies in astronomical time series analysis. II-Statistical aspects of spectral analysis of unevenly spaced data, Astrophys. J. 263, 835 (1983).

[43] J. W. Zhao, B. H. Sun, I. Tanihata, S. Terashima, L. H. Zhu, A. Enomoto, D. Nagae, T. Nishimura, S. Omika, A. Ozawa et al., Reaching time resolution of less than 10 ps with plastic scintillation detectors, Nucl. Instrum. Methods Phys. Res., Sect. A 823, 41 (2016).

[44] M. Wang, G. Audi, F. G. Kondev, W. Huang, S. Naimi, and $\mathrm{X}$. Xu, The AME2016 atomic mass evaluation (II). Tables, graphs and references, Chin. Phys. C 41, 030003 (2017).

[45] J. C. Yang, J. W. Xia, G. Q. Xiao, H. S. Xu, H. W. Zhao, X. H. Zhou, X. W. Ma, Y. He, L. Z. Ma, D. Q. Gao et al., High Intensity heavy ion Accelerator Facility (HIAF) in China,
Nucl. Instrum. Methods Phys. Res., Sect. B 317, 263 (2013).

[46] J. Liu, J. C. Yang, J. W. Xia, D. Y. Yin, G. D. Shen, P. Li, B. Wu, S. Ruan, H. Zhao, G. Wang et al., Transverse impedances and collective instabilities in a heavy ion accelerator, Phys. Rev. Accel. Beams 21, 064403 (2018).

[47] B. Wu, J. C. Yang, J. W. Xia, X. L. Yan, X. J. Hu, L. J. Mao, L. N. Sheng, J. X. Wu, D. Y. Yin, W. P. Chai et al., The design of the spectrometer ring at the HIAF, Nucl. Instrum. Methods Phys. Res., Sect. A 881, 27 (2018).

[48] Y. A. Litvinov and F. Bosch, Beta decay of highly charged ions, Rep. Prog. Phys. 74, 016301 (2011).

[49] P. M. Walker, Y. A. Litvinov, and H. Geissel, The ILIMA project at FAIR, Int. J. Mass Spectrom. 349-350, 247 (2013).

[50] A. Dolinskii, S. Litvinov, M. Steck, and H. Weick, Study of the mass resolving power in the CR storage ring operated as a TOF spectrometer, Nucl. Instrum. Methods Phys. Res., Sect. A 574, 207 (2007). 